\title{
BMJ A case-control study examining Open whether neurological deficits and PTSD in combat veterans are related to episodes of mild TBI
}

\author{
Robert Louis Ruff, ${ }^{1,2,3}$ Ronald George Riechers II, ${ }^{1,2,3}$ Xiao-Feng Wang, ${ }^{4}$ \\ Traci Piero, ${ }^{3}$ Suzanne Smith Ruff ${ }^{3,5}$
}

To cite: Ruff RL, Riechers RG II, Wang X-F, et al. A case-control study examining whether neurological deficits and PTSD in combat veterans are related to episodes of mild TBI. BMJ Open 2012;2: e000312. doi:10.1136/ bmjopen-2011-000312

- Prepublication history and additional appendices for this paper are available online. To view these files please visit the journal online (http://dx. doi.org/10.1136/ bmjopen-2011-000312)

Received 22 August 2011 Accepted 22 February 2012

This final article is available for use under the terms of the Creative Commons Attribution Non-Commercial 2.0 Licence; see http://bmjopen.bmj.com

For numbered affiliations see end of article.

\section{ABSTRACT}

Background: Mild traumatic brain injury (mTBI) is a common injury among military personnel serving in Iraq or Afghanistan. The impact of repeated episodes of combat mTBI is unknown.

Objective: To evaluate relationships among mTBI, post-traumatic stress disorder (PTSD) and neurological deficits (NDS) in US veterans who served in Iraq or Afghanistan.

Methods: This was a case-control study. From 2091 veterans screened for traumatic brain injury, the authors studied 126 who sustained mTBI with one or more episodes of loss of consciousness (LOC) in combat. Comparison groups: 21 combat veterans who had definite or possible episodes of $\mathrm{mTBI}$ without LOC and 21 veterans who sustained MTBI with LOC as civilians.

Results: Among combat veterans with mTBI, $52 \%$ had NDs, $66 \%$ had PTSD and 50\% had PTSD and an ND. Impaired olfaction was the most common ND, found in 65 veterans. The prevalence of an ND or PTSD correlated with the number of mTBI exposures with LOC. The prevalence of an ND or PTSD was $>90 \%$ for more than five episodes of LOC. Severity of PTSD and impairment of olfaction increased with number of LOC episodes. The prevalence of an ND for the 34 combat veterans with one episode of LOC $(4 / 34=11.8 \%)$ was similar to that of the 21 veterans of similar age and educational background who sustained civilian mTBI with one episode of LOC $(2 / 21=9.5 \%$, p-NS).

Conclusions: Impaired olfaction was the most frequently recognised ND. Repeated episodes of combat $\mathrm{MTBI}$ were associated with increased likelihood of PTSD and an ND. Combat setting may not increase the likelihood of an ND. Two possible connections between mTBI and PTSD are (1) that circumstances leading to combat $\mathrm{mTBI}$ likely involve severe psychological trauma and (2) that altered cerebral functioning following $\mathrm{mTBI}$ may increase the likelihood that a traumatic event results in PTSD.

\section{ARTICLE SUMMARY}

Article focus

- Case-control study of mTBI associated with LOC among US veterans who were deployed to Iraq or Afghanistan during Operations Iraqi Freedom and Enduring Freedom.

- Three study groups: (1) 126 veterans who had mTBI with LOC, (2) 21 OIF/OEF veterans who did not suffer mTBI with LOC and (3) 21 veterans who sustained $\mathrm{mTBI}$ with LOC in a civilian setting.

- Evaluated NDs including a quantitative test of olfaction, PTSD with severity assessed using the PCL-M instrument and a cognitive function using the Montreal Cognitive Assessment Test.

Key messages

- Olfaction was a sensitive test for neurological injury associated with mTBI with LOC.

- More episodes of MTBI with LOC were associated with higher prevalence rates of NDs or of PTSD.

- The severity of PTSD and extent of olfactory impairment increased with the number of episodes of LOC; cognitive function performance was inversely related to the number of episodes of LOC.

Strengths and limitations of this study

- Case-control study of US combat veterans with mTBI who were assessed for NDs, PTSD and cognitive function.

- Subjects and comparison groups had detailed assessments for NDs, and combat veterans were also assessed for PTSD.

- The findings should be relevant to other groups of military personnel with combat mTBI.

- The neurological examination was not blinded.

- The selection of veterans in this study may be biased because veterans who do not have health issues may not seek care from the Department of Veterans Affairs.

- The comparison groups were small.

- The findings in veterans with mTBI with LOC may not apply to people with $\mathrm{mTBI}$ without LOC. 


\section{INTRODUCTION}

Traumatic brain injury (TBI) is a major worldwide cause of death and disability. The worldwide incidence is about 10 million cases per year. ${ }^{1}$ This is likely an underestimate of the true incidence because cases of mild traumatic brain injury (mTBI) are often unreported. In the USA, TBI is the most common neurological diagnosis leading to treatment in emergency/urgent care facilities with about 1 million cases of TBI yearly and 50000 deaths per year. ${ }^{2}$ Most studies of TBI focus on moderate to severe TBI, and there are relatively few studies of TBI incurred in military combat. mTBI, or concussion, is a common military injury among NATO troops serving in Iraq and Afghanistan (OIF/OEF). ${ }^{3}$ When US Army troops were evaluated after a deployment to Iraq, between $15 \%$ and $20 \%$ reported at least one episode of TBI, predominantly mTBI. ${ }^{4-6}$ In a study of US National Guard soldiers who were deployed to Iraq, when queried 1 month before the end of a deployment, $9.2 \%$ of soldiers reported an mTBI during the deployment and $22.0 \%$ reported an $\mathrm{mTBI}$ during the deployment when queried 1 year later. ${ }^{7}$ The cause for the delay in selfrecognition of $\mathrm{mTBI}$ is uncertain. Several factors may increase reporting of mTBI after deployment. 'Over time, retrospective recall of combat events and history of concussion/mTBI may be influenced by current symptoms of distress, attributions about current psychosocial difficulties and secondary gain., ${ }^{7}$ Alternatively, mTBI may be under-reported during a deployment. 'While in theater, soldiers may minimize reports of concussion/ mTBI history to remain with their units, live up to perceived expectations of superiors and peers, and ensure health concerns do not delay return home during demobilization.'

Combat produces psychologically traumatic events; hence, stress reactions including post-traumatic stress disorder (PTSD) frequently accompany mTBI. ${ }^{4}$ The presence of some post-concussion symptoms such as impaired memory appears to correlate more strongly with the presence of PTSD rather than with mTBI. ${ }^{4} 7$ However, post-traumatic headaches seem to be more strongly associated with mTBI. ${ }^{4}$ The prevalence of neurological deficits (NDs) associated with combat mTBI and the relationships among ND, PTSD and number of episodes of mTBI are unknown.

In this case-controlled observational study, we screened 2091 OIF/OEF veterans and identified 126 veterans who had one or more episode of combat mTBI associated with loss of consciousness (LOC). The research questions we examined were (1) what are the most frequently recognised NDs that can be identified during an examination performed in a clinic setting? (2) Do associations exist between the episodes of TBI and NDs or PTSD? We evaluated veterans for NDs and PTSD and correlated outcomes with LOC episodes. We had two comparison groups. To evaluate whether episodes of mTBI with LOC differed from mTBI without LOC, we compared the findings in combat veterans who experienced mTBI episodes with LOC to combat veterans who did not have any LOC episodes. To consider if a combat setting influenced the likelihood of a veteran having residual NDs, we compared the findings in combat veterans who experienced mTBI episodes with LOC to veterans who suffered mTBI with LOC as civilians. The veterans in the comparison groups had the same testing as the veterans who had combat mTBI with LOC, which led to the smaller sizes of the comparison groups relative to the study group.

\section{MATERIAL AND METHODS}

This was a case-controlled observational study of a cohort of OIF/OEF veterans with mTBI that began as a Neurology Service Quality Assurance Monitor of the evaluation of OIF/OEF veterans with mTBI. We collected information from the veterans in an unblinded, but uniform and prospective, manner. Veterans did not sign consent forms. Data were collected at the Louis Stokes Department of Veterans Affairs Medical Center in Cleveland (CVAMC), which is a regional Polytrauma Center that addresses the needs of OIF/OEF veterans for most of the State of Ohio. The CVAMC Institutional Review Board reviewed the data in this report, approved waiver of HIPAA authorisation, granted a waiver of informed consent and approved submission of the data in this manuscript for publication.

There were three study groups: (1) $126 \mathrm{OIF} / \mathrm{OEF}$ veterans with one or more episode of LOC due to combat mTBI, (2) $21 \mathrm{OIF} / \mathrm{OEF}$ veterans who had no episodes of LOC and (3) 21 veterans who sustained an episode of mTBI with LOC as civilians.

\section{mTBI criteria}

mTBI criteria was an episode of TBI with $\mathrm{LOC}<30 \mathrm{~min}$, duration of any alteration in consciousness (AOC) following the TBI was $<24 \mathrm{~h}$ and post-traumatic amnesia (PTA) was $<24$ h. ${ }^{9}$ We focused on veterans who had LOC because TBI depended upon historical recall. In a combat setting, it can be difficult to distinguish AOC from conflict-induced changes in arousal or emotion.

We defined combat mTBI as occurring (1) during engagement with enemy or (2) associated with an explosion from an enemy device such as mortar/artillery shell, rocket-propelled grenade, improvised explosive device or bomb. Non-combat TBI occurred during a deployment without enemy engagement or an explosion.

\section{Subject selection-OIF/OEF veterans}

The OIF/OEF veterans were individuals who sought care from the Veterans Health Administration of the United States (VHA) often for issues not related to TBI such as treatment of musculoskeletal pain or choosing VHA to be their personal health resource. In addition, many individuals had mental health issues such as depression and PTSD. OIF/OEF veterans were screened for TBI using a three-level sequential process. The first screening 
step was a four question screening tool that was administered to all OIF/OEF veterans treated by VHA. ${ }^{10}$ For those who confirm OEF or OIF deployment and do not have a prior diagnosis of TBI, the instrument proceeds using four sequential question sets. The initial screen is negative if a person responds negatively to any question set. If the veteran affirms one or more possible answer in each section, the screen is positive. The four sections are (1) events that could heighten the risk of TBI such as explosion exposure; (2) immediate symptoms following the event including LOC, AOC or PTA; (3) new or worsening symptoms following the event and (4) current symptoms that are consistent with TBI. The natural history of mTBI is that most individuals with civilian mTBI not associated with an explosion have resolution of symptoms within 6 months. ${ }^{11}$ The recovery pattern of combat mTBI associated with an explosion is that a greater fraction of individuals can have persisting postconcussion symptoms following mTBI; however, these individuals also have PTSD that is likely contributing to the persistence of symptoms. ${ }^{12}$ The symptoms in the screen included alterations in cognition, behaviour, motor or sensory function, balance or coordination and the presence of pain including headache. During a 24-month period between 2006 and 2008, 2091 OIF/ OEF veterans were screened with the four question TBI screening tool ${ }^{10}$ and 385 screened positive (18.4\%). The veterans who were screened were veterans who sought healthcare with VHA. Figure 1 is a flow chart indicating the steps in identification of study subjects. We have a breakdown of the reasons that veterans screened negative during a 6-month interval within the entire 24-month window that we considered. During that

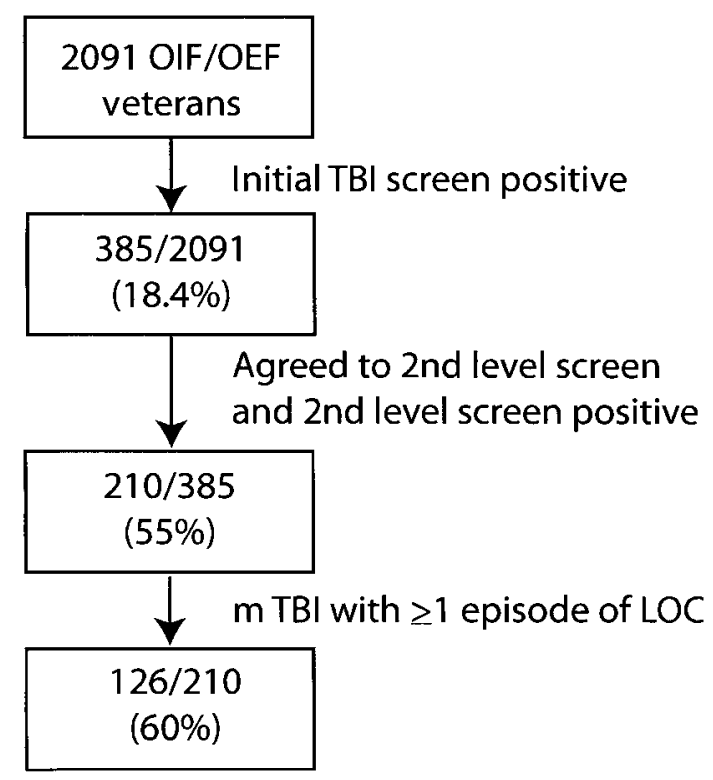

Figure 1 Flow chart of the selection of veterans with episodes of mild traumatic brain injury (mTBI) associated with loss of consciousness (LOC). 6-month period, $2 \%$ of veterans who screened negative indicated no exposure to an event associated with TBI (first screen section), $29 \%$ indicated that they did not have symptoms following exposure to an event that could produce TBI (second screen section), 31\% indicated that they did not develop new symptoms following a TBI (third screen section) and $38 \%$ indicated that they had symptoms following TBI but that they did not currently have symptoms associated with TBI (fourth screen section). Veterans identified on the first screen were encouraged to undergo a second-level evaluation including an interview and physical examination; 350 had second-level screening $(90.9 \%)$. The second-level screening was consistent with TBI in $210(60 \%)$ and 178 agreed to additional assessment $(84.8 \%)$. Among the 178 veterans, 65 were initially interviewed by a nurse practitioner (TP), trained in TBI assessment, to determine if they had sustained an episode of TBI; she found that $42(64.6 \%)$ had histories of mTBI with LOC. The first author (RLR) performed a detailed assessment of the $113 / 178$ veterans identified on the second-level screen and not evaluated by the nurse practitioner plus the 42 identified by the nurse practitioner, 155 veterans in total. This assessment typically took $2.5 \mathrm{~h}$. It consisted of an interview, a structured neurological examination and a cognitive assessment test (described below). Veterans were asked, using open-ended questions, to describe what happened when they may have had TBI. Periods when the veteran was dazed or confused were considered as possible episodes of AOC. Periods when veterans could not remember what happened or had gaps in their memories for events before or after episodes of head trauma were considered to be PTA episodes. For each episode, LOC referred to a period when someone at the trauma scene observed the veteran to be unresponsive or the veteran did not move when prompted or nudged. If there were no observers, veterans could indicate an episode of LOC by stating that they clearly aroused or 'woke-up' following head trauma.

The 155 veterans evaluated by RLR were divided into two groups. One group contained 126 veterans who had one or more episode of combat mTBI with LOC. The second group contained 21 combat veterans who did not sustain LOC: 11 veterans with $\mathrm{mTBI}$ in a combat setting, five veterans who had $\mathrm{mTBI}$ in a non-combat setting and five veterans who did not have a definite episode of TBI. In the last su-group of five veterans, each veteran had at least one episode of exposure to an explosion that was associated with a behavioural change that RLR interpreted as possible but not definite episodes of AOC. The episodes in these five veterans may have been changes in arousal or emotion associated with combat rather than episodes of AOC. Thus, in this group of 21 veterans, 16 had episodes of mTBI associated with AOC and five had episodes that may have been mTBI. In comparisons, we considered both the group of 16 veterans who had definite episodes of mTBI without LOC and the larger 
group of 21 veterans that included the five veterans who probably had mTBI without LOC. We excluded eight veterans who had moderate or penetrating TBI. The size of the comparison group of combat veterans with mTBI without LOC reflects the small fraction of veterans who did not have LOC among the 155 veterans examined by RLR. The 155 veterans examined by RLR had undergone two prior screening evaluations that indicated the veterans had mTBI with persisting residual symptoms such as headache. Seventy-nine/126 veterans (62.7\%) who had combat mTBI with LOC and 12/21 $(57.1 \%)$ who did not have LOC were National Guard or Reservists.

\section{Veterans with civilian $\mathrm{mTBI}$}

We evaluated 36 veterans for TBI with LOC in a civilian environment. These veterans did not report prior episodes of TBI, including during military service. They were evaluated within 3 months of the mTBI. Thus, each veteran in this group experienced the first episode of mTBI as a civilian. We excluded 12 veterans because they did not have TBI-six, had moderate TBI-three, or because they were more than 65 years old-three. The age exclusion was because we did not expect to have combat veterans $>65$ years old. Etiologies of mTBI were motor vehicle accident (MVA) - eight, work-related TBI- seven (five falls), sporting - three, non-work falltwo, and assault-one.

\section{Neurological examination}

Neurological examination was previously described and included 50 scored elements (online appendix 1). ${ }^{13}$ This examination included a screening test of cognitive function; cranial nerve testing; motor function testing, including assessment of tone, muscle bulk, strength and symmetry of movements; sensory function including sensation in the extremities and face and extinction of sensation with simultaneous stimulations; extremity coordination and precision of movement; stability of standing and gait. We included a 12-item quantitative olfactory test (Brief Smell Identification Test; Sensonics, Haddon Heights, New Jersey, USA, http:// www.sensonics.com). ${ }^{14}$ Each scent was evoked in a standardised manner, and the veteran identified the scent from a list of possibilities. The olfactory test had age-adjusted normal values. ${ }^{15}$ Normal olfaction were scores $\geq 7 / 12$ for $<55$ years old and $\geq 5 / 12$ for $\geq 55$ years old. We elicited no histories of impaired olfaction before OIF/OEF deployments. We chose the Montreal Cognitive Assessment (MOCA) Test to measure cognitive function because it is widely used within the Veterans Health Administration, it does not have a licensing fee and it has been used for repeated measures. ${ }^{16}$ Performance on the MOCA is influenced by age and other demographic factors. ${ }^{17}$ The median ages of the subjects in the three study groups indicate that the expected normal range of scores would be $26-30 .{ }^{17}$ RLR administered the MOCA after obtaining the TBI history.
Every combat veteran was assessed for PTSD

Every combat veteran was assessed for PTSD using the Primary Care PTSD Screen (PC-PTSD). ${ }^{18}$ The initial screen had four questions about PTSD symptoms. If a veteran acknowledged any three items, the screen was considered positive. Veterans who screened positive were assessed using a 17-item National Center for PTSD checklist for symptoms of military PTSD (PCL-M), ${ }^{19}$ and the veterans were referred for further evaluation by a mental health professional qualified to diagnose PTSD. The mental health professional would often employ other PTSD evaluation instruments including the Mississippi Scale for Combat Related Posttraumatic Stress Disorder ${ }^{20}$ Each veteran had an interview to assess for the presence and severity of PTSD. Mental health professionals chose an interview instrument based upon their familiarity with the instrument and suitability to assessment of PTSD associated with military service. The most common interview instrument used was the Clinician Administered PTSD Scale. ${ }^{21}$ Mental health professionals initiated treatment plans based upon assessment of the strengths of each veteran and the likeliness that a veteran would benefit from different treatment options. ${ }^{22}$

PTSD diagnosis satisfied the American Psychiatric Association's Diagnostic and Statistical Manual of Mental Disorders, 4th edition, criteria of at least one intrusion item (eg, PCL-M questions 1-5), three avoidance (eg, PCL-M questions 6-12) and two hyper-arousal symptoms (eg, PCL-M questions 13-17). ${ }^{19}$ Symptoms rated as 'moderately severe' or greater (responses three through five) were counted as present. In addition, the individual had to have substantial distress indicated by a PCL-M score of $>50 .{ }^{19}$

\section{Statistical methods}

Independent variables were (1) mTBI episodes with LOC, (2) smoking history and (3) OIF/OEF deployment duration. Dependent variables were (1) NDs, (2) olfactory score, (3) presence of PTSD, (4) PCL-M score and (5) MOCA score. The primary outcome measures were two categorical variables: the presence of NDs and presence of PTSD. The secondary outcome measures were olfaction scores, PCL-M scores and MOCA scores. We studied the relationship between the prevalence of ND/PTSD and the number of LOC episodes. We also studied the relationship between the number of LOC episodes and MOCA scores, PCL-M scores or olfactory test results.

We compared the MOCA scores using the two-sided $\mathrm{t}$ test and olfaction scores using both the two-sided test and the nonparametric Wilcoxon rank-sum test. ${ }^{23}$ Outcome frequencies were analysed by Pearson's $\chi^{2}$ test procedure or Fisher's exact test. In table 2, we evaluated the correlations between episodes of LOC and the presence of an ND or PTSD using generalised Spearman's rank correlation coefficients. ${ }^{24}$ The correlations of MOCA, PCL-M and olfactory test scores and the numbers of episodes of LOC were evaluated using 
Table 1 Frequencies of abnormalities on neurological testing, PTSD and MOCA scores for combat veterans with combat $\mathrm{mTBI}$, combat veterans who did not have LOC and veterans who sustained $\mathrm{mTBI}$ in a civilian setting

\begin{tabular}{|c|c|c|c|c|}
\hline Patient group & $\begin{array}{l}\text { Deficit on } \\
\text { neurological } \\
\text { examination }\end{array}$ & $\begin{array}{l}\text { Deficits on neurological } \\
\text { examination other than } \\
\text { olfaction }\end{array}$ & PTSD & MOCA scores \\
\hline $\begin{array}{l}\text { Combat veterans with } \\
\text { mTBI }(n=126)\end{array}$ & $65(52 \%)$ & $29(23 \%)$ & $83(66 \%)$ & $25.1 \pm 0.18$ \\
\hline $\begin{array}{l}\text { Combat veterans without } \\
\text { LOC }(n=21)^{*}\end{array}$ & $0(0 \%), p<0.001$ & $0(0 \%), p<0.001$ & $2(9.5 \%), p<0.001$ & $28.8 \pm 0.29, p<0.001$ \\
\hline $\begin{array}{l}\text { Combat veterans with } \\
\text { definite mTBI without } \\
\text { LOC }(n=16)^{\star}\end{array}$ & $0(0 \%), p<0.001$ & $0(0 \%), p<0.001$ & $1(6.25 \%), p<0.001$ & $28.9 \pm 0.32, p<0.001$ \\
\hline $\begin{array}{l}\text { Veterans with civilian } \\
\text { mTBI }(n=21)\end{array}$ & $2(9.5 \%), p<0.001$ & $1(4.8 \%), p<0.001$ & $1(4.8 \%), p<0.001$ & $28.4 \pm 0.23, p<0.001$ \\
\hline
\end{tabular}

Kendall's rank correlation analysis. ${ }^{23}$ Correlations of MOCA scores with PCL-M and olfaction scores were obtained using both Kendall's rank correlation analysis and Pearson's product-moment correlation coefficient. ${ }^{23}$ The Bonferroni method was used to correct for multiple comparisons. ${ }^{25}$ The Bonferroni $p$ value ( $p_{\text {Bonferroni }}$ ) adjusts the raw $p$ value $\left(p_{\text {raw }}\right)$ for the number of times that a hypothesis is tested (number of comparisons $=\mathrm{m}), \mathrm{p}_{\text {Bonferroni }}=\mathrm{m} \cdot \mathrm{p}_{\text {raw }}$. If the adjusted $\mathrm{p}$ value exceeds 1 , it is set to 1 . The Bonferroni test is conservative but always controls the familywise error rate. The probability values shown in tables 1 and 2 were corrected using the Bonferroni method. Values are presented as means $\pm \mathrm{SE}$ of the mean.

\section{RESULTS}

The veteran groups had similar demographic features. The mean ages were combat veterans with LOC $(n=126)-29.2 \pm 2.6$ years (range $20-62$ years), combat veterans without LOC $(n=21)-30.0 \pm 1.6$ years (range
21-48 years), and veterans with civilian mTBI $(n=21)-$ $35.1 \pm 2.2$ years (range 21-53 years). The combat veterans older than 40 years were National Guard or Reservists. The percentages of women/high school graduates/college graduates were combat veterans with LOC-7.9/100/8.7, combat veterans without LOC $-9.5 / 100 / 5$, and veterans with civilian mTBI-9.5/ $100 / 9.5$. The two groups of combat veterans had similar mean numbers of deployments / total deployment lengths: combat veterans with $\mathrm{LOC}-1.43 \pm 0.06$ deployments (range $1-4$ deployments) $/ 81.4 \pm 3.2$ weeks (range 40-208 weeks), and combat veterans without LOC$1.76 \pm 0.25$ deployments (range $1-4$ deployments)/ $90.8 \pm 12.0$ weeks (range 40-208 weeks).

Veterans with combat-acquired mTBI with LOC had low MOCA scores and high frequencies of an ND and the presence of PTSD (table 1). ND were reduced olfaction-65, impaired balance-14, abnormal eye movements - 13, motor asymmetry-two, and sensory change-two. Twenty-nine veterans had more than one ND. Among the 65 veterans with NDs, 36 (55\%) had

Table 2 Correlations between the number of episodes of LOC and the presence of PTSD, presence of an ND, MOCA scores, PCL-M scores or olfactory test results for combat veterans

\begin{tabular}{lll}
\hline Correlation of episodes of mTBI with outcomes and correlation & mTBI associated with combat \\
\cline { 2 - 2 } of PCL-M and olfaction tests scores with MOCA scores & Correlation coefficient & p Value \\
\hline LOC vs ND (including olfaction) & 0.314 & $<0.01$ \\
LOC vs ND (excluding olfaction) & 0.254 & $<0.01$ \\
LOC vs PTSD & 0.405 & $<0.01$ \\
LOC vs MOCA score & -0.226 & $<0.01$ \\
LOC vs PCL-M score & -0.665 & $<0.01$ \\
LOC vs olfaction score & $-0.620(-0.765)$ & $<0.01$ \\
MOCA score vs PCL-M score & $0.00497(0.0926)$ & $<0.001$ for both \\
MOCA score vs olfaction score & -0.194 & NS for both \\
PCL-M vs olfaction & NS \\
\hline Kendall's rank correlation analysis coefficients are shown in the table with Pearson's product-moment correlation coefficients shown in \\
parentheses. \\
LOC, loss of consciousness; MOCA, Montreal Cognitive Assessment; mTBI, mild traumatic brain injury; ND, neurological deficit; PTSD, post- \\
traumatic stress disorder.
\end{tabular}


only impaired olfaction. Impaired balance was detected using the Romberg test. ${ }^{26}$ The most frequently recognised abnormal eye movement was saccadic dysmetria (12 individuals), with one individual having asymmetric horizontal saccade velocity. ${ }^{27}$ Motor asymmetry was detected with arm-rolling and upper extremity drift that were both present in two subjects. ${ }^{28}$ The two veterans with sensory changes had extinction on simultaneous stimulation.

Half of the 126 veterans with combat mTBI had PTSD and an ND, 21 (17\%) had PTSD without an ND, five (4\%) had an ND without PTSD and 37 (29\%) did not have PTSD or an ND. Among the 63 veterans with PTSD and an ND, mean MOCA scores were 24.0 \pm 0.26 and they were deployed $41.3 \pm 2.03$ weeks, whereas veterans who did not have PTSD or an ND had higher MOCA scores $(26.5 \pm 0.23, \mathrm{p}<0.001)$ but similar durations of deployment, $38.8 \pm 3.09$ weeks.

In contrast, 21 combat veterans who did not have LOC episodes had no NDs, a lower frequency of PTSD and higher MOCA scores (table 1). Their olfaction scores were higher than the 61 veterans who sustained mTBI with LOC without an ND, $11.1 \pm 0.17$ vs $10.6 \pm 0.34$ $(p<0.01)$. The comparison group of 16 veterans who had definite episodes of mTBI without LOC also had a lower frequency of PTSD and higher MOCA scores (table 1). The olfaction scores of the group of 16 veterans were also higher than the scores of the veterans who had mTBI with LOC without an ND, 11.25 \pm 0.11 $(\mathrm{p}<0.01)$.

Because the most frequent ND was impaired olfaction, we considered other causes of olfactory impairment: smoking, ${ }^{29}$ upper respiratory infection ${ }^{30}$ and nasal sinus disease. ${ }^{30}$ Smoking frequencies among combat veterans with or without NDs were similar $(38.4 \%$ vs $39.34 \%$, p-NS). Four veterans had upper respiratory congestion at the time of olfactory testing (1) or within the prior month (3). When retested $6-8$ weeks later, the olfactory score for three veterans did not change. The score of the veteran with a symptomatic upper respiratory infection when initially tested improved to normal. The normal score was used for analysis. The frequencies of histories of nasal sinus disease were similar for veterans who had impaired olfaction $(7.7 \%)$ versus those with normal olfaction scores $(8.2 \%)$. Head imaging studies were not routinely done; however, 34 veterans with an ND $(52.3 \%)$ and 31 veterans without an ND $(50.8 \%)$ had an MRI or CT scan that visualised the sinuses. Four imaging studies for veterans with an ND (12\%) and four for veterans without an ND (13\%) revealed unilateral sinus disease. No individual had bilateral sinus findings.

Veterans with combat mTBI experienced 380 episodes of LOC, mean of 3.02 \pm 0.20 episodes of LOC per veteran (range of 1-8 episodes of LOC for a veteran, online appendix 2 shows the numbers of veterans with specific numbers of episodes of LOC). Most episodes of LOC were associated with an explosion. Explosions occurred with 304 episodes of LOC (80\%). Each veteran with
LOC had at least one episode of LOC associated with an explosion. Factors contributing to combat mTBI were improvised explosive device-261, MVA-197, rocket-propelled grenade-52, artillery/mortar-32, bomb-20, and blunt trauma not associated with explosion or MVA-10. Combat mTBI could have several contributing factors. Explosions were often associated with an MVA. Five veterans reported one LOC in a noncombat setting caused by fall, fight, MVA, sports injury and striking a doorframe. Veterans also experienced episodes of AOC without LOC (only AOC).

The presence of an ND (including or excluding veterans whose only ND was impaired olfaction), the presence of PTSD, MOCA, PCL-M and olfaction scores were related to the number of episodes of LOC (table 2, figure 2). The mean MOCA score for one episode of combat-acquired LOC was $26.1 \pm 0.34 \quad(\mathrm{n}=34)$ and $25.3 \pm 0.36$ for two episodes of LOC $(p<0.05)$. Veterans with five or more episodes of LOC had mean MOCA scores of $24.2 \pm 0.41 \quad(p<0.05$ compared with two episodes of LOC). In contrast, the strengths of associations were weakened by considering mTBI episodes with only AOC. Associations of episodes of LOC + only AOC episodes were ND-0.131, PTSD-0.262, and MOCA -0.085 (all $\mathrm{p}<0.001$ ). There were no associations of NDs, PTSD or MOCA scores with episodes of only AOC.

For veterans with PTSD, MOCA scores correlated inversely with PTSD severity measured with PCL-M scores (table 2, figure 3A). However, there was no correlation between MOCA and olfaction scores for veterans with an ND (table 2, figure 3B) or veterans with normal olfaction (data not shown). For veterans with PTSD and impaired olfaction, PCL-M scores were not correlated with olfaction scores (table 2, figure 4).

We compared OIF/OEF veterans with a group of 21 veterans who sustained $\mathrm{mTBI}$ with LOC in a civilian setting to evaluate the contribution of a combat setting (table 1). Neither group reported episodes of TBI before military service. Veterans with civilian mTBI reported no TBI during military service. All veterans with civilian mTBI had one episode of LOC. Intervals from the last TBI differed. Veterans with combat TBI were seen 122.6 \pm 4.4 weeks after the last TBI (range 40-212 weeks). This time reflected the time OIF/OEF veterans spent in the military before they were discharged and the time it took for the OIF/OEF veterans to obtain care through the CVAMC. We evaluated veterans with civilian TBI $8.38 \pm 0.53$ weeks (range $4-12$ weeks, $p<0.001$ ) after mTBI. Veterans with civilian mTBI had fewer NDs, lower frequency of PTSD and higher MOCA scores compared with those with combat mTBI (table 1). However, when veterans with civilian mTBI were compared with the 34 veterans who had one episode of combat mTBI, the two groups had similar frequencies of NDs of $4 / 34(11.8 \%)$ for combat mTBI and $2 / 21(9.5 \%, \mathrm{p}-\mathrm{NS})$ for civilian mTBI. The two veterans with civilian mTBI and initial NDs did not have 
Figure 2 Effect of number of episodes of loss of consciousness (LOC) on outcomes for veterans with combat-acquired mild traumatic brain injury: (A) olfaction scores, (B) post-traumatic stress disorder (PTSD) severity as measured by the PCL-M score, (C) score on the Montreal Cognitive Assessment (MOCA) Test and (D) the prevalence of abnormalities on neurological examination (unfilled circles) or PTSD (filled squares). The correlation coefficients for the association between the number of episodes of LOC and the olfaction scores, PCL-M scores and MOCA score are shown in table 2. The straight lines in A, B and C correspond to Kendall's rank correlation analysis coefficients in table 2.
A

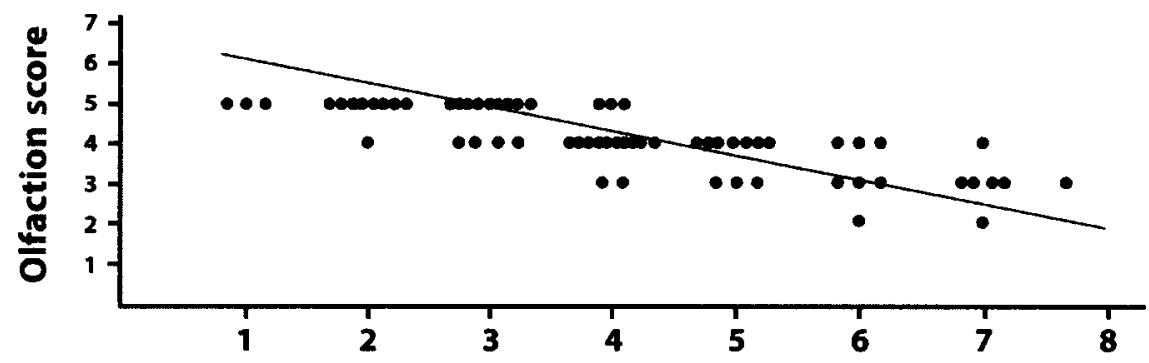

B

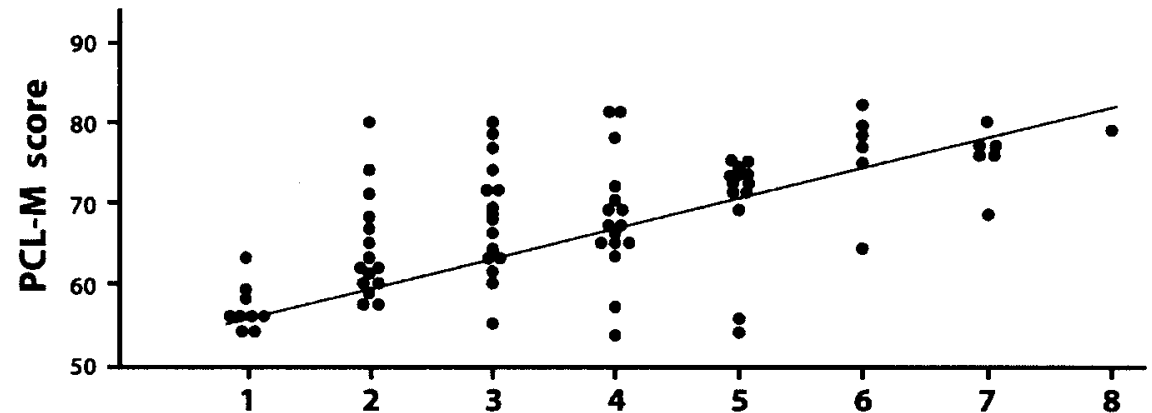

C

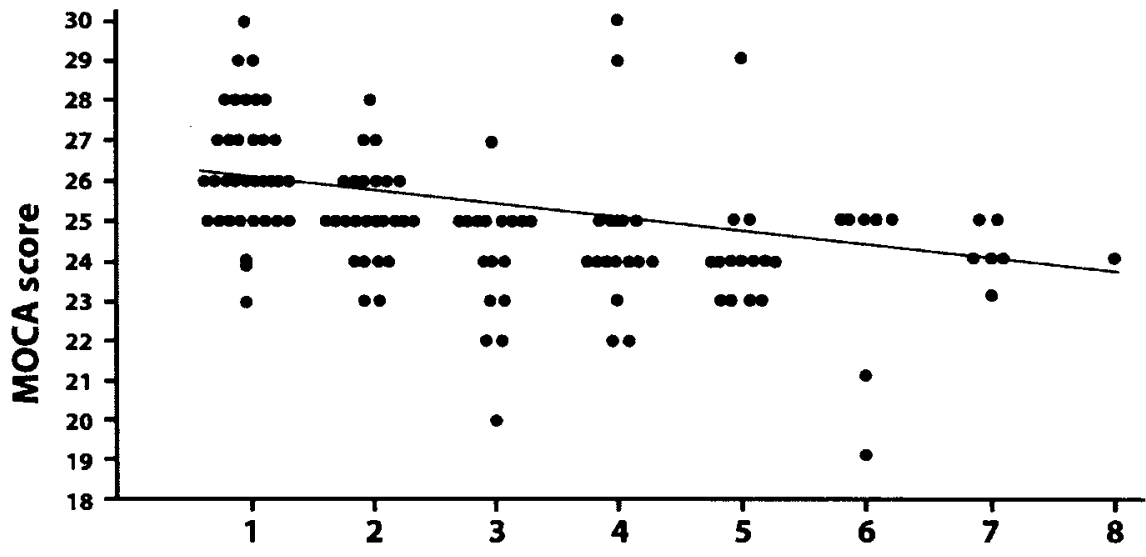

D

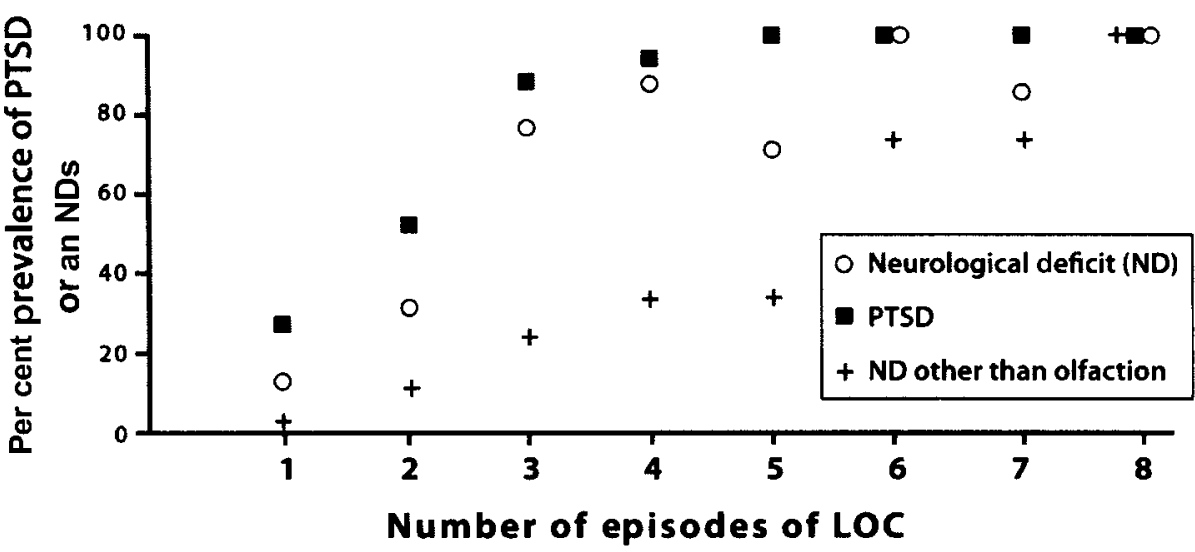

any NDs when re-examined 2 years later, an interval between TBI and evaluation comparable to the interval for combat mTBI. Due to the small sizes of the groups of veterans with $\mathrm{mTBI}$ and one episode of LOC, the ND prevalence values remained not significantly different. MOCA scores for veterans with civilian mTBI (table 1) were higher than for veterans with one episode of combat-associated LOC, 26.1 $\pm 0.34, \mathrm{p}<0.001$. PTSD was more common for combat veterans with one episode of LOC $(32.4 \%$ vs $4.8 \%, p=0.0194)$. 
Figure 3 Association of the scores on the Montreal Cognitive Assessment (MOCA) Test with (A) post-traumatic stress disorder (PTSD) severity as measured by the PCL-M score for veterans with PTSD or (B) olfaction scores for veterans with neurological deficits including impaired olfaction. The correlation coefficients for the association between the MOCA scores and the PCL-M scores are shown in table 2. There was no association between MOCA scores and olfaction scores. The straight line in A corresponds to Kendall's rank correlation analysis coefficient in table 2.
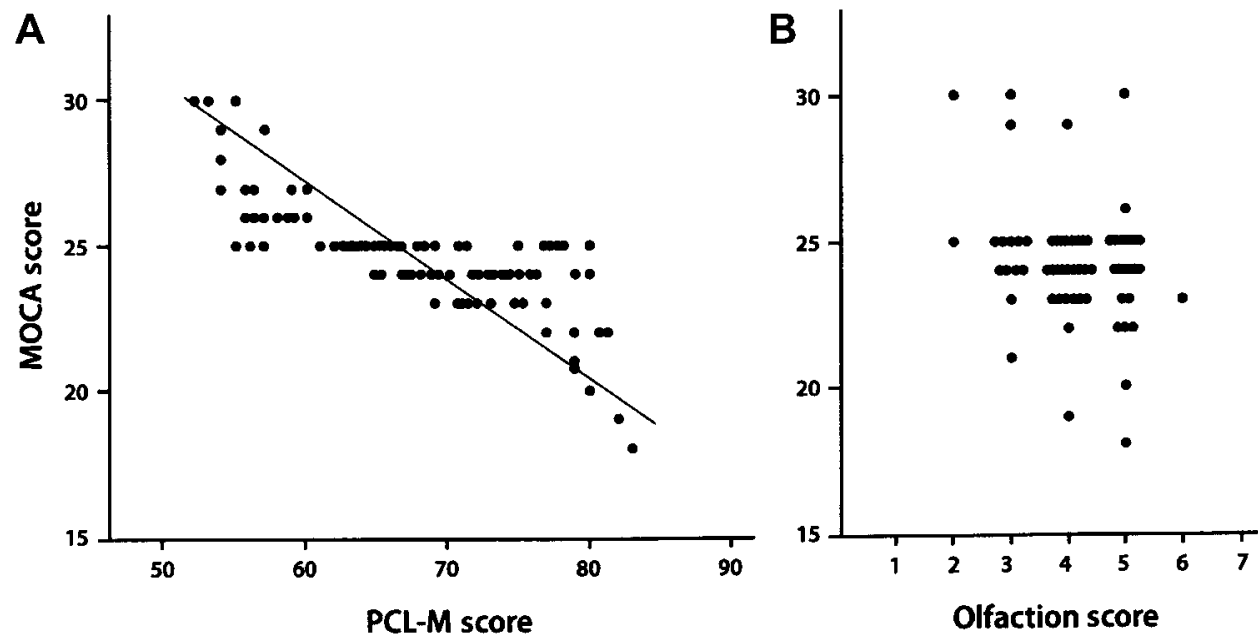

addition, PCL-M scores, extent of olfactory impairment and reduction in MOCA scores increased with the number of episodes of LOC (figure 2). The most frequently recognised ND was impaired olfaction followed by impaired balance and abnormal saccades. Testing for olfaction using a 'scratch and sniff' instrument is easy to do in a clinic setting, has been validated for different subject groups and is amenable to a variety of settings including a combat environment. ${ }^{14}{ }^{15} \mathrm{We}$ advocate incorporating olfaction testing into neurological examinations for TBI. Among the items in the 50 element neurological examination, the other elements, aside from olfaction, that indicated neurological dysfunction were the Romberg test, observation of saccades, asymmetric arm rolling/arm drift and consistent unilateral sensory extinction on simultaneous light touch stimulation of both upper extremities. About $40 \%$ of civilians with TBI have impaired balance ${ }^{31}$ or impaired eye movements. ${ }^{32}$ However, specialised testing environments are needed to detect the subtle changes in balance or eye movements produced by mTBI. These assessments are not done in a clinic setting. Olfactory testing is the most sensitive indicator of persisting injury

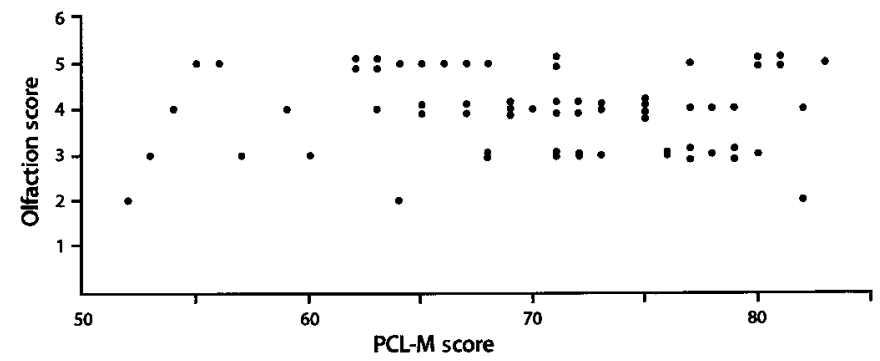

Figure 4 Association of the PCL-M and olfaction test scores for veterans with both NDs and post-traumatic stress disorder. The correlation coefficient for the association between the PCL-M scores and olfaction scores is shown in table 2. There was no significant association between PCL-M scores and olfaction scores. following TBI that can be done in a clinic setting and is a good test for remote TBI because olfaction usually does not recover after TBI. ${ }^{33}$

We found the strongest associations between outcomes (NDs, PTSD and MOCA scores) and episodes of LOC. Veterans who experienced episodes of LOC had MOCA scores that were mildly reduced compared with those who did not experience episodes of LOC. The absolute differences in MOCA scores between different groupings of the veterans in this study were small, even though the differences may have been statistically significant. The high frequency of episodes of LOC associated with a blast prevented us from being able to discern differences between episodes of LOC associated with explosions and episodes of LOC not associated with explosions. All the veterans who had an episode of LOC had at least one episode of LOC associated with an explosion. When episodes of AOC as well as LOC were considered, the correlations were weaker and there were no correlations for episodes of AOC alone. We could not distinguish prevalence differences for NDs following civilian versus combat mTBI, but the numbers of veterans with one episode of LOC were small.

Several biases may have influenced this study. Episodes of TBI were historical. In some instances, veterans may have underestimated the duration of LOC so that some episodes of mTBI may have really been episodes of moderate TBI. Neurological examinations were not blinded. However, the olfaction test and the MOCA are objective tests that reduce the likelihood that RLR influenced veterans' performance. Due to the nature of the armed conflicts in the Middle East with repeated deployments for US combat troops and because episodes of mTBI were not likely to remove one from combat responsibilities, there could be a long time between the last episode of mTBI and VA assessment. This was not a random sample of veterans who sustained mTBI in OIF/OEF. Veterans who sustained mTBI without persisting problems would be less likely to seek treatment from the CVAMC, and veterans who had 
complaints that might be attributed to a prior mTBI might misremember that an episode of trauma included mTBI when there was no TBI. The associations of NDs with episodes of mTBI suggest that the recalled episodes of mTBI were associated with cerebral injury. There were relatively few subjects in the comparison group of combat veterans who had mTBI without LOC. The size of this group was not representative of the expected fraction of combatants with mTBI without LOC. In two studies of military personnel who returned from deployment in Iraq or Afghanistan, between 26\% and $32 \%$ of those who reported an episode of TBI reported having LOC. ${ }^{44}$ However, the comparison groups were only used for the data shown in table 1. The data presented in the other table and figures do not involve the comparison groups. The presence of combat veterans up to 62 years of age may be due to two factors: (1) the delay between end of military service and enrolment for VHA care and (2) that $63 \%$ of those with LOC were National Guard or Reservists.

The MOCA is a cognitive screening test. Performance on the MOCA decreases with age and extent of education. The subjects in this study all had at least 12 years of formal education and were $<65$ years of age so that age would not be expected to have had a prominent effect on their MOCA performance. ${ }^{17}$ As a cognitive function test, the MOCA is not as sensitive as detailed intelligence testing, but detailed intelligence testing would have taken longer times to administer. The MOCA has been used as a cognitive screening test for neurological disorders such as Parkinson's disease and stroke, and the MOCA is more sensitive than some other cognitive screening tests such as the Mini-Mental State Examination. ${ }^{35} 36$

Veterans with combat mTBI frequently complain of impaired cognition. ${ }^{8}$ Did PTSD or NDs have the strongest influence on veterans' performance? PCL-M scores correlated with cognitive performance on the MOCA. Our data indicating that PTSD severity influenced cognitive performance is consistent with prior suggestions that PTSD influenced post-deployment symptoms including poor cognition. ${ }^{4}$ The lack of association between MOCA and olfaction scores may indicate that the MOCA test is not sensitive to damage to orbital frontal cortex associated with impaired olfaction. ${ }^{37}$ We found that deployment duration and the number of deployments were similar for veterans who did or did not suffer NDs and PTSD, which argues against NDs or PTSD resulting from factors that may be related to deployment durations such as exposure to a possible Middle East pathogen.

Was impaired olfaction caused by mTBI or other factors? Olfactory function can be impaired by smoking, sinus disease and inhalation of irritants. ${ }^{29} 3038$ We found no increase in smoking prevalence among veterans with impaired olfaction or differences in the history or presence of sinus disease. Exposure to pollutants and smoke, which may be present in a combat environment, produces $\sim 12 \%$ decrease in olfaction test scores, ${ }^{38}$ which would not explain our findings. PTSD does not compromise olfaction, ${ }^{39}$ and we found that olfactory impairment did not correlate with PTSD severity. Therefore, we believe that reduced olfaction scores were likely due to repeated episodes of mTBI.

Quantitative tests of olfaction have not been used previously in studies of combat TBI. In one civilian study of mTBI, 22\% of subjects had hyposmia and $4 \%$ had anosmia after one mTBI. ${ }^{40}$ We found that $12 \%$ of veterans with combat mTBI and one episode of LOC had hyposmia. Civilian subjects may have had a higher prevalence of impaired olfaction because they were evaluated sooner. Injured olfactory nerve fibres can recover over time leading to recovery of olfaction during the first year after TBI. ${ }^{41}$ Consequently, the veterans that we evaluated who had impaired olfaction, likely had permanent olfaction impairments. These olfaction impairments were usually not recognised by the veterans.

We believe that the olfaction deficits did not result in any functional limitations. The importance of the olfaction deficits was that they were markers of cerebral injury, specifically frontal lobe injury. The following text describes the relationships between TBI and impaired olfaction and how cerebral damage including injury to the ventromedial frontal lobes can enhance the likelihood that a psychologically traumatic event leads to the genesis of PTSD.

TBI usually impairs olfaction by shearing the olfactory nerves traversing the cribiform plate, bruising the orbital frontal cortex or both. ${ }^{37}$ A study of US military personnel who sustained an episode of combat TBI, predominantly mTBI, in OIF/OEF found that $29 \%$ of subjects had white matter lesions in two or more areas of interest. ${ }^{42}$ The areas of injury were orbital frontal cortex, cingulum and middle cerebellar peduncles. The finding of orbital frontal cortex injury associated with combat mTBI provides a structural cerebral correlation for our observation of impaired olfaction.

Civilian TBI studies indicate that neurological dysfunction correlates with the number of TBI events. ${ }^{43} 44$ Recovery from mTBI is slower following repeated mTBIs. ${ }^{45}$ Repeated sports concussions compromise cerebral electrical activity and metabolism. ${ }^{46}{ }^{47}$ Dementia and cerebral degeneration may be delayed consequences of repeated concussions. ${ }^{48}{ }^{49}$ Our observation that ND prevalence increased with combat mTBI episodes is consistent with observations from civilian concussions.

The increase in PTSD prevalence that we observed with LOC episodes may be due to several factors. The events causing LOC may have produced sufficient psychological trauma to induce PTSD. The presence of mTBI may have increased the likelihood that a psychologically traumatic event resulted in PTSD. ${ }^{50}$ A study of the development of anxiety disorders in children following TBI found that $8.5 \%$ of children developed anxiety disorders, usually PTSD, within 6 months of the 
TBI. ${ }^{51}$ Children with mTBI had the greatest likelihood of developing PTSD. An Australian study of civilian trauma reported that the prevalence of PTSD following mTBI was $6 \%$, which was about 1.9-fold higher than following trauma without TBI. ${ }^{52}$ PTSD and mTBI are associated in combat personnel. About $40 \%$ of military personnel and veterans with combat-acquired mTBI have PTSD. ${ }^{4} 8$ Among soldiers recently deployed in Iraq who experienced an episode of LOC due to mTBI, $44 \%$ had PTSD compared with $16 \%$ for soldiers with other injuries and $9 \%$ for uninjured soldiers. ${ }^{4}$

'Data suggest that PTSD is associated with over activation of the amygdala due to a lack of inhibitory control by ventromedial prefrontal cortex, as well as deficient hippocampal function. ${ }^{53}$ The areas of brain injury identified by functional imaging in PTSD including ventromedial frontal lobes and medial temporal lobes $^{54} 55$ are included within the areas damaged in mTBI. ${ }^{42}$ 56-58

In this study, impaired olfaction was the most frequently recognised ND. To the extent that impaired olfaction is a marker for injury to orbital frontal cortex, impaired olfaction may be a flag for a cerebral injury that can facilitate the development of PTSD. The association of impaired olfaction with the presence of PTSD and PTSD severity is consistent with impaired olfaction being a marker for damage to orbital frontal cortex with reduced inhibition of the amygdala enabling anxiety and exaggerated fear responses. ${ }^{53}$

Pre-existing subtle ('soft') ND may increase the risk of developing PTSD. ${ }^{59}$ The 45 neurological soft signs assessed in Vietnam veterans were similar to the 50 items in the neurological examinations performed in this study but did not include olfaction testing. In studies of monozygotic twins where one twin was in combat and the other not, among twins pairs where one had combatassociated PTSD, both twins had a higher prevalence of neurological soft signs ${ }^{59}$ or grey matter abnormalities in the right hippocampus, pregenual anterior cingulate cortex and left and right insular cortex ${ }^{60}$ than the twins where the combat-exposed twin did not have PTSD. The twin studies suggested that subtle genetically based NDs can potentiate the genesis of PTSD. This is, however, distinct from the findings of the Vietnam Head Injury Program (VHIP) ${ }^{53}$ where veterans with combat penetrating TBIs were classified based on lesion location. The prevalence of PTSD was compared across groups. Patients with amygdala or ventromedial frontal injuries had reduced prevalence of PTSD. The apparent contradiction between the twins and VHIP studies may be due in part to differences in the severity of the injuries. Subjects in the twin studies had subtle performance and imaging deficits compared with the penetrating injuries in the VHIP study. Mild injury may potentiate PTSD genesis by slightly disrupting normal interactions among the amygdala, ventromedial frontal cortex and hippocampus, whereas more severe injury may prevent "the 'super-normal' levels of fear/anxiety that define PTSD." ${ }^{53}$ In childhood TBI, development of PTSD and other anxiety disorders correlated with MRIidentified damage to the superior frontal gyrus, anterior frontal white matter and orbital frontal cortex. ${ }^{51}$ Overall, the risk of an individual with combat TBI developing PTSD may be higher for mTBI compared with severe TBI. ${ }^{61}$ Children with mTBI had a greater likelihood of developing PTSD than children who had severe TBI. ${ }^{51}$ In addition, penetrating injuries may have been more focal and less likely to have the more diffuse axonal effects of closed head injury. We found that PTSD severity was not associated with the severity of olfaction impairment. Perhaps the presence of injury to orbital cortex predisposes to PTSD, but severity of cortex injury is not the primary factor controlling PTSD severity. Our observation that the severity of olfaction impairment was related to the number of LOC events suggests that impaired olfaction was related to combat mTBI rather than being pre-existing deficits.

Several factors can enhance or reduce the likelihood that a psychologically traumatic event results in development of anxiety disorders including PTSD. For example, psychological resiliency, a supportive social environment and higher levels of intelligence and education may reduce the likelihood of an individual developing PTSD. ${ }^{22} 62$ This study suggests that mTBI in a combat setting may enhance the likelihood of an individual developing PTSD. Additional studies are needed to support or refute the suggestion from this study that mTBI increases the likelihood that combat trauma leads to PTSD.

There is a tendency to attribute physical symptoms after deployment to PTSD rather than to mTBI. ${ }^{4}$ Future studies of combat TBI will determine the extent to which our findings of increased prevalence of PTSD and NDs with episodes of LOC generalise to other populations of military personnel and in other settings. Given the results detailed in this study suggesting a relationship between mTBI and PTSD, perhaps the focus of future studies should shift from ascribing cognitive deficits and physical symptoms to one diagnosis versus another to understanding the impact of repeated combat mTBI on development of PTSD.

\section{Author affiliations \\ ${ }^{1}$ Neurology Service, Louis Stokes Cleveland Department of Veterans Affairs Medical Center, Cleveland, Ohio, USA \\ ${ }^{2}$ Department of Neurology, Case Western Reserve University, Cleveland, Ohio, USA \\ ${ }^{3}$ Polytrauma System of Care, Louis Stokes Cleveland Department of Veterans Affairs Medical Center, Cleveland, Ohio, USA \\ ${ }^{4}$ Department of Quantitative Health Sciences, The Cleveland Clinic, Cleveland, Ohio, USA \\ ${ }^{5}$ Psychology Service, Louis Stokes Cleveland Department of Veterans Affairs} Medical Center, Cleveland, Ohio, USA

Contributors RLR examined the subjects, collected data, did data analysis and wrote the manuscript. RGR II edited the manuscript, assisted with data interpretation and wrote portions of the discussion. X-FW did the statistical analysis and edited the portions of the manuscript related to statistical 
analysis. TP examined subjects and edited the manuscript. SSR examined subjects, assisted with study design and edited the manuscript.

Funding RLR is the Medical Director of the Functional Electrical Stimulation Center of Cleveland, which is supported by a Center of Excellence Award from the Rehabilitation Research and Development Service of the Office of Research and Development of the Department of Veterans Affairs. X-FW was retained as a biostatistician for the Functional Electrical Stimulation Center of Cleveland. His involvement on this project was supported by funding from the Department of Veterans Affairs through the Center of Excellence Award to the Functional Electrical Stimulation Center of Cleveland. The work in this manuscript was supported through funding for the care of veterans from the Veterans Health Administration. RLR and SSR and RGR and TP are salaried clinicians of the Veterans Health Administration.

Competing interests There were no other actual or potential conflicts of interest for the authors that could have inappropriately influenced the present work. Subjects and their medical records were treated in accordance with internal review board approved policies and procedures. Standard professional and ethical guidelines were upheld during the research study and manuscript preparation. The views expressed in this article do not necessarily reflect those of the Veterans Health Administration of the Department of Veterans Affairs of the USA or the USA government.

Ethics approval Ethical approval was provided by Institutional Review Board of the Cleveland VA Medical Center.

Provenance and peer review Not commissioned; externally peer reviewed.

Data sharing statement The data used in this study are available in the form of a subject de-identified spreadsheet that can be obtained by a written request to RLR, robert.ruff1@va.gov.

\section{REFERENCES}

1. Hyder AA, Wunderlich CA, Puvanachandra P, et al. The impact of traumatic brain injuries: a global perspective. NeuroRehabilitation 2007;22:341-53.

2. Binder S, Corrigan JD, Langlois JA. The public health approach to traumatic brain injury: an overview of CDC's research and programs. J Head Trauma Rehabil 2005;20:189-95.

3. Warden DL. Military TBI during the Iraq and Afghanistan wars. J Head Trauma Rehabil 2006;21:398-402.

4. Hoge CW, McGurk D, Thomas JL, et al. Mild traumatic brain injury in U.S. soldiers returning from Iraq. N Engl J Med 2008;358:453-63.

5. Terrio $\mathrm{H}$, Brenner LA, Ivins BJ, et al. Traumatic brain injury screening: preliminary findings in a US Army Brigade Combat Team. J Head Trauma Rehabil 2009;24:14-23.

6. Theeler BJ, Flynn FG, Erickson JC. Headaches after concussion in US soldiers returning from Iraq or Afghanistan. Headache 2010;50:1262-72.

7. Polusny MA, Kehle SM, Nelson NW, et al. Longitudinal effects of mild traumatic brain injury and posttraumatic stress disorder comorbidity on postdeployment outcomes in national guard soldiers deployed to Iraq. Arch Gen Psychiatry 2011;68:79-89.

8. Gironda RJ, Clark ME, Ruff RL, et al. Traumatic brain injury, polytrauma, and pain: challenges and treatment strategies for the polytrauma rehabilitation network. Rehabil Psychol 2009;54:247-58.

9. Malec JF, Brown AW, Leibson CL, et al. The mayo classification system for traumatic brain injury severity. J Neurotrauma 2007;24:1417-24.

10. Donnelly KT, Donnelly JP, Dunnam M, et al. Reliability, sensitivity, and specificity of the VA traumatic brain injury screening tool. J Head Trauma Rehabil 2011;26:439-53.

11. McCrea M, Iverson GL, McAllister TW, et al. An integrated review of recovery after mild traumatic brain injury (MTBI): implications for clinical management. Clin Neuropsychol 2009;23:1368-90.

12. Burgess $P$, Sullivent EE, Sasser SM, et al. Managing traumatic brain injury secondary to explosions. J Emerg Trauma Shock 2010;3:164-72.

13. Ruff RL, Ruff SS, Wang XF. Headaches among veterans of Operations Iraqi Freedom and Enduring Freedom with mild traumatic brain injury associated with exposures to explosions. J Rehabil Res Dev 2008;45:941-53.

14. Doty RL, Marcus A, Lee WW. Development of the 12-item CrossCultural Smell Identification Test (CC-SIT). Laryngoscope 1996;106:353-6.

15. Doty RL. Olfaction. Annu Rev Psychol 2001;52:423-52.
16. Nasreddine ZS, Phillips NA, Bédirian V, et al. The Montreal Cognitive Assessment, MOCA: a brief screening tool for mild cognitive impairment. J Am Geriatr Soc 2005;53:695-9.

17. Rossetti HC, Lacritz LH, Cullum CM, et al. Normative data for the Montreal Cognitive Assessment (MoCA) in a population-based sample. Neurology 2011;77:1272-5.

18. Prins A, Ouimette P, Kimerling R, et al. The primary care PTSD screen (PC-PTSD): development and operating characteristics. Prim Care Psychiatr 2003;9:9-14.

19. Department of Veterans Affairs. National Center for PTSD: Using the PTSD Checklist (PCL). 2010. http://www.ptsd.va.gov/professional/ pages/assessments/ptsd-checklist.asp

20. Keane TM, Caddell JM, Taylor KL. Mississippi Scale for CombatRelated Posttraumatic Stress Disorder: three studies in reliability and validity. J Consult Clin Psychol 1988;56:85-90.

21. Blake DD, Weathers FW, Nagy LM, et al. The development of a clinician-administered PTSD scale. J Traumatic Stress 1995;8:75-90.

22. Peterson AL, Luethcke CA, Borah EV, et al. Assessment and treatment of combat-related PTSD in returning war veterans. J Clin Psychol Med Settings 2011;18:164-75.

23. Hollander M, Wolfe DA. Nonparametric Statistical Methods. New York: John Wiley \& Sons, 1973:185-94.

24. Harrell FE. Regression Modeling Strategies With Applications to Linear Models, Logistic Regression, and Survival Analysis. New York: Springer, 2001.

25. Benjamini $Y$, Hochberg $Y$. Controlling the false discovery rate: a practical and powerful approach to multiple testing. J Roy Stat Soc B 1995;57:289-300.

26. Khasnis A, Gokula RM. Romberg's test. J Postgrad Med 2003:49:169-72.

27. Leigh RJ, Zee DS. The Neurology of Eye Movements. 4th edn. Oxford: Oxford University Press, 2006

28. Sawyer RN Jr, Hanna JP, Ruff RL, et al. Asymetry of forearm rolling as a sign of unilateral cerebral dysfunction. Neurology 1993;43:1595-8.

29. Frye RE, Schwartz BS, Doty RL. Dose-related effects of cigarette smoking on olfactory function. JAMA 1990;263:1233-6.

30. Deems DA, Doty RL, Settle $\mathrm{G}$, et al. Smell and taste disorders, a study of 750 patients from the University of Pennsylvania Smell and Taste Center. Arch Otolaryngol Head Neck Surg 1991;117:519-28.

31. Pickett TC, Radfar-Baublitz LS, McDonald SD, et al. Objectively assessing balance deficits after TBI: role of computerized posturography. J Rehabil Res Dev 2007;44:983-90.

32. Kraus MF, Little DM, Donnell AJ, et al. Oculomotor function in chronic traumatic brain injury. Cogn Behav Neurol 2007;20:170-8.

33. London B, Nabet B, Fisher AR, et al. Predictors of prognosis in patients with olfactory disturbance. Ann Neurol 2008;63:159-66.

34. Schwab KA, Ivins B, Cramer G, et al. Screening for traumatic brain injury in troops returning from deployment in Afghanistan and Iraq initial investigation of the usefulness of a short screening tool for traumatic brain injury. J Head Trauma Rehabil 2007;22:377-89.

35. Hoops S, Nazem S, Siderowf AD, et al. Validity of the MoCA and MMSE in the detection of $\mathrm{MCl}$ and dementia in Parkinson disease. Neurology 2009;73:1738-45.

36. Pendlebury ST, Cuthbertson FC, Welch SJ, et al. Underestimation of cognitive impairment by Mini-Mental State Examination versus the Montreal Cognitive Assessment in patients with transient ischemic attack and stroke: a population-based study. Stroke 2010;41:1290-3

37. Kern RC, Quinn B, Rosseau G, et al. Post-traumatic olfactory dysfunction. Laryngoscope 2000;110:2106-9.

38. Altman KW, Desai SC, Moline J, et al. Odor identification ability and self-reported upper respiratory symptoms in workers at the post-9/11 World Trade Center site. Int Arch Occup Environ Health 2011;84:131-7.

39. Vermetten E, Schmahl C, Southwick SM, et al. Positron tomographic emission study of olfactory induced emotional recall in veterans with and without combat-related posttraumatic stress disorder. Psychopharmacol Bull 2007;40:8-30.

40. de Kruijk JR, Leffers $P$, Menheere PP, et al. Olfactory function after mild traumatic brain injury. Brain Inj 2003;17:73-8.

41. Doty RL, Yousem DM, Pham LT, et al. Olfactory dysfunction in patients with head trauma. Arch Neurol 1997;54:1131-40.

42. Mac Donald CL, Johnson AM, Cooper D, et al. Detection of blastrelated traumatic brain injury in U.S. military personnel. $N$ Engl J Med 2011;364:2091-100.

43. Guskiewicz KM, McCrea M, Marshall SW, et al. Cumulative effects associated with recurrent concussion in collegiate football players: the NCAA Concussion Study. JAMA 2003;290:2549-55.

44. Wall SE, Williams WH, Cartwright-Hatton $\mathrm{S}$, et al. Neuropsychological dysfunction following repeat concussions in jockeys. J Neurol Neurosurg Psychiatry 2006;77:518-20. 
45. Slobounov S, Slobounov E, Sebastianelli W, et al. Differential rate of recovery in athletes after first and second concussion episodes. Neurosurgery 2007;61:338-44; discussion 344.

46. De Beaumont L, Brisson B, Lassonde $\mathrm{M}$, et al. Long-term electrophysiological changes in athletes with a history of multiple concussions. Brain Inj 2007:21:631-44.

47. Henry LC, Tremblay S, Boulanger $\mathrm{Y}$, et al. Neurometabolic changes in the acute phase after sports concussions correlate with symptom severity. J Neurotrauma 2010;27:65-76.

48. Guskiewicz KM, Marshall SW, Bailes J, et al. Association between recurrent concussion and late-life cognitive impairment in retired professional football players. Neurosurgery 2005;57:719-26.

49. McKee AC, Cantu RC, Nowinski CJ, et al. Chronic traumatic encephalopathy in athletes: progressive tauopathy after repetitive head injury. J Neuropathol Exp Neurol 2009;68:709-35.

50. Ruff RL, Riechers RG, Ruff SS. Relationships between mild traumatic brain injury sustained in combat and post-traumatic stress disorder. F1000 Med Rep 2010;2:64. The electronic version of this article can be found at. http://f1000.com/reports/medicine/content/2/64

51. Max JE, Keatley E, Wilde EA, et al. Anxiety disorders in children and adolescents in the first six months after traumatic brain injury. J Neuropsychiatry Clin Neurosci 2011;23:29-39.

52. Bryant RA, O'Donnell ML, Creamer M, et al. The psychiatric sequelae of traumatic injury. Am J Psychiatry 2010;167:312-20.

53. Koenigs M, Huey ED, Raymont V, et al. Focal brain damage protects against post-traumatic stress disorder in combat veterans. Nat Neurosci 2008;11:232-7.

54. Whalley MG, Rugg MD, Smith AP, et al. Incidental retrieval of emotional contexts in post-traumatic stress disorder and depression: an fMRI study. Brain Cogn 2009;69:98-107.
55. Sailer U, Robinson S, Fischmeister FP, et al. Altered reward processing in the nucleus accumbens and mesial prefrontal cortex of patients with posttraumatic stress disorder. Neuropsychologia 2008;46:2836-44.

56. Topal NB, Hakyemez B, Erdogan C, et al. MR imaging in the detection of diffuse axonal injury with mild traumatic brain injury. Neurol Res 2008;30:974-8.

57. Lo C, Shifteh K, Gold T, et al. Diffusion tensor imaging abnormalities in patients with mild traumatic brain injury and neurocognitive impairment. J Comput Assist Tomogr 2009;33:293-7.

58. Peskind ER, Petrie EC, Cross DJ, et al. Cerebrocerebellar hypometabolism associated with repetitive blast exposure mild traumatic brain injury in 12 Iraq War Veterans with persistent postconcussive symptoms. Neuroimage 2011;54(Suppl 1):S76-82.

59. Gurvits TV, Metzger LJ, Lasko NB, et al. Subtle neurologic compromise as a vulnerability factor for combat-related posttraumatic stress disorder: results of a twin study. Arch Gen Psychiatry 2006;63:571-6.

60. Kasai K, Yamasue H, Gilbertson MW, et al. Evidence for acquired pregenual anterior cingulate gray matter loss from a twin study of combat-related post-traumatic stress disorder. Biol Psychiatry 2008;63:550-6.

61. Carlson K, Kehle S, Meis L, et al. The Assessment and Treatment of Individuals with History of Traumatic Brain Injury and Post-Traumatic Stress Disorder: A Systematic Review of the Evidence. Washington DC: Department of Veterans Affairs Health Services Research \& Development Service, 2009.

62. King DW, King LA, Foy DW, et al. Posttraumatic stress disorder in a national sample of female and male Vietnam veterans: risk factors, war-zone stressors, and resilience-recovery variables. $J$ Abnorm Psychol 1999;108:164-70. 